\title{
Conductivity of a 2D electron crystal over liquid helium in the region of coupled electron-ripplon resonances
}

\author{
Vitaliy Syvokon and Yuri Kovdrya \\ B. Verkin Institute for Low Temperature Physics and Engineering of the National Academy of \\ Sciences of Ukraine, 47 Lenin Ave., Kharkov 61103, Ukraine \\ E-mail: sivokon@ilt.kharkov.ua
}

\begin{abstract}
The dependence of the coupled electron-ripplon oscillations spectrum of a $2 \mathrm{D}$ electron crystal over liquid helium on the holding electric field is studied at a temperature much lower than the temperature of melting. An analysis of the spectrum near the main resonance $(0,1)$ allowed finding both the real and imaginary components of the crystal conductivity. The imaginary part of the conductivity is shown to be in good agreement with theoretical estimates. At the same time, additional theoretical and experimental studies are necessary to clear up the possible reasons for energy losses in the electron crystal.
\end{abstract}

PACS: 67.70.+n, 73.21.-b, 73.63.-b

There exist two interconnected aspects of interest in the ordered electron system over the liquid helium surface - electron or Wigner crystal (EC). The first is related to the interaction of the $2 \mathrm{D}$ solid with the surface of liquid helium and the properties of the helium surface, and the second is connected with the properties of the 2D solid itself, including the features of the $2 \mathrm{D}$ crystal melting.

As a result of crystallization the coupled electron-ripplon oscillations which are due to the interaction between plasma modes of the electron system and capillary waves can be excited in the EC [1,2]. Many aspects of the coupled oscillations are well studied. The resonance frequencies of such oscillations, calculated within the self-consistent theory [3], agree rather well with the experimental data [4]. The resonances in EC are a reliable tool for studing the surface properties of liquid helium. The method of resonances in EC was employed to measure of the surface tension coefficients in ${ }^{3} \mathrm{He}^{-4} \mathrm{He}$ mixtures [5].

However, important features of the interaction of the EC with the helium surface have not been adequately studied. In particular, it is not very clear what process is responsible for the energy losses in the EC. The movement of the EC along the helium surface was studied theoretically for uniform [6] and nonuniform [7] electric fields along the surface (leading fields). The energy loss of from the EC was supposed to be a result of attenuation of the capillary waves. It follows from the experimental data [8] on the attenua- tion of the coupled electron-ripplon oscillations that the losses are at least two order of magnitude higher than the result predicted by the capillary wave attenuation in a comparable frequency range.

The aim of present work is study the attenuation of the coupled oscillations in the EC. Measurements are performed at various values of the holding electric field, which is one of the essential parameters of the electron-surface interaction. An electron crystal with a surface density of $6.4 \cdot 10^{8} \mathrm{~cm}^{-2}$ (the melting at temperature $T=0.56 \mathrm{~K}$ ) was studied at a temperature of $83 \mathrm{mK}$. The holding field varied from $E_{\perp}=$ $=640 \mathrm{~V} / \mathrm{cm}$, corresponding to complete screening of the holding potential by the surface electrons, to $E_{\perp}=$ $=1180 \mathrm{~V} / \mathrm{cm}$.

For the measurements an experimental cell of Corbino geometry was employed. It consisted of a horizontally placed plane capacitor with plates of $2.7 \mathrm{~cm}$ diameter and gap of $0.2 \mathrm{~cm}$. The helium surface with the electrons over it was placed approximately in the middle of the gap. The cell was adjusted at $T \sim 1.5 \mathrm{~K}$ to have uniform thickness of the helium layer above the bottom capacitor plate. Accuracy of the adjustment was better than $20 \mu \mathrm{m}$. The positive electric potential was applied to the bottom plate to form the holding field. The upper capacitor plate consisted of ring measuring electrodes. The ac voltage was applied to the input (exciting) electrode. The voltage caused an ac electric field in the electron layer plane and electron density variations. The variations spread out along the 
layer and induced a current in the output electrode. The relation between the voltage at the input electrode and current in the output electrode reflects the properties (complex conductivity $\sigma$ ) of the electron layer. To estimate $\sigma$ from the output-input relation the solution of Maxwell's equations for the cell was used. The solution, the experimental cell, and the measurement procedure were described in detail in Ref. 9.

The experiment started from the placing of electrons on the helium surface at $T \sim 1.5 \mathrm{~K}$ until complete screening of the holding potential was reached. After that the cell was cooled down to $T=83$ $\mathrm{mK}$. During the cooling the crystallization in the electron layer was observed, and the crystallization temperature was used to determine the electron density.

A voltage with an amplitude of $1 \mathrm{mV}$ and a frequency in the range $1-20 \mathrm{MHz}$ was applied to the input electrode, and the current induced in the output electrode was measured. In the range $1-20 \mathrm{MHz}$ the resonance features in the EC were observed. The value of exciting amplitude of $1 \mathrm{mV}$ was a compromise between the necessity of having a suitable signal-to-noise ratio and of remaining in the linear measurement mode in which there is no dependence of the EC properties on the input signal. The frequency and relative amplitude of main resonance $(0,1)$ were measured at input amplitudes of $0.3,0.5,1$, and $2 \mathrm{mV}$ and led to the conclusion that the $1 \mathrm{mV}$ amplitude corresponds rather to the linear mode border, although there was no noticeable difference between the frequencies and relative amplitude of the $(0,1)$ mode at the $0.3,0.5$, and $1 \mathrm{mV}$ signal levels.

The primary experimental data are shown in Fig. 1. Figure 1, $a$ illustrates the frequency dependence of the induced current amplitude at the various holding fields. Here $f=\omega / 2 \pi$. On the vertical axis the voltage on the input resistance of the measuring circuit pre-amplifier is plotted. Figure $1, b$ shows the phase shift between the input and output signals. As one can see in Fig. 1, $a$, the response of the cell to the exciting signal has the main resonance and a few less pronounced resonances. It is also seen that as $E_{\perp}$ increases, the resonances except for the main are suppressed. The modes $(0,1),(0,2)$, and $(0,3)$ calculated according to he theory [9] are shown as triangles. The experimental frequencies of the mode $(0,1)$ are in good accordance with the theoretical ones at all $E_{\perp}$. There is good enough accordance for the mode $(0,2)$ at $E_{\perp}=1060 \mathrm{~V}$ and $E_{\perp}=1180 \mathrm{~V}$. The distinctive features at frequencies around $8-11 \mathrm{MHz}$ at the lowest $E_{\perp}$ can be interpreted as the modes $(0,2)$ and $(0,3)$ shifted noticeably to the lower frequency range. Probably such a shift could be a result of nonlinearities in the EC response.
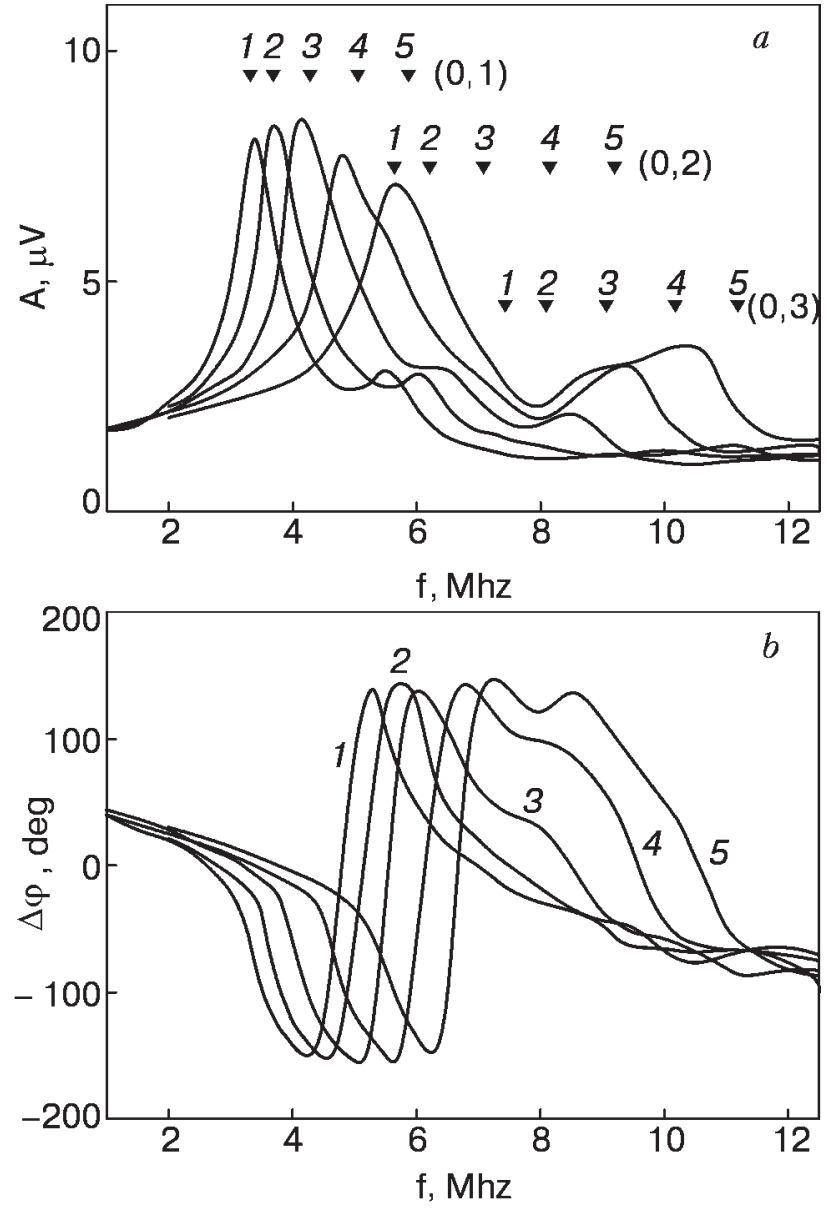

Fig. 1. Amplitude ( $a$ ) and phase shift (b) of the response of an electron crystal with a surface density of $6.4 \cdot 10^{-8} \mathrm{~cm}^{-2}$ at temperature $T=83 \mathrm{mK}$ on the exciting ac voltage for frequences of the holding fields $E_{\perp}, \mathrm{V} / \mathrm{cm}$ : 1150 (1); 1000 (2); 850 (3); 705 (4); 590 (5). The triangles correspond to the calculated frequencies of the resonant modes $(0,1),(0,2),(0,3)$.

The experimental values of the frequency $f_{\text {res }}$ and the width $\Delta f_{\text {res }}$ of the resonance $(0,1)$ allow one to calculate the real and imaginary parts of $\sigma$, using the solution of Maxwell's equations for the cell [9]. The solution consists of two expressions reflecting relation between measured values of $G_{1}$ and $G_{2}$ in the expression for the output current $J$ and components of the inverse conductivity of the electron layer $\chi_{1}=\operatorname{Re} \sigma_{\text {eff }}^{-1}$ and $\chi_{2}=\operatorname{Im} \sigma_{\text {eff }}^{-1}$ :

$$
J=\left(G_{1}+i \omega G_{2}\right) V_{0} \mathrm{e}^{i \omega t},
$$

where $V_{0}$ is exciting voltage amplitude.

Components of the inverse conductivity of the electron layer $\chi_{1}$ and $\chi_{2}$ can be calculated not only from $f_{\text {res }}$ and $\Delta f_{\text {res }}$ but from any pair of lineary independent functions of $\chi_{1}$ and $\chi_{2}$ which are capable of being measured. 

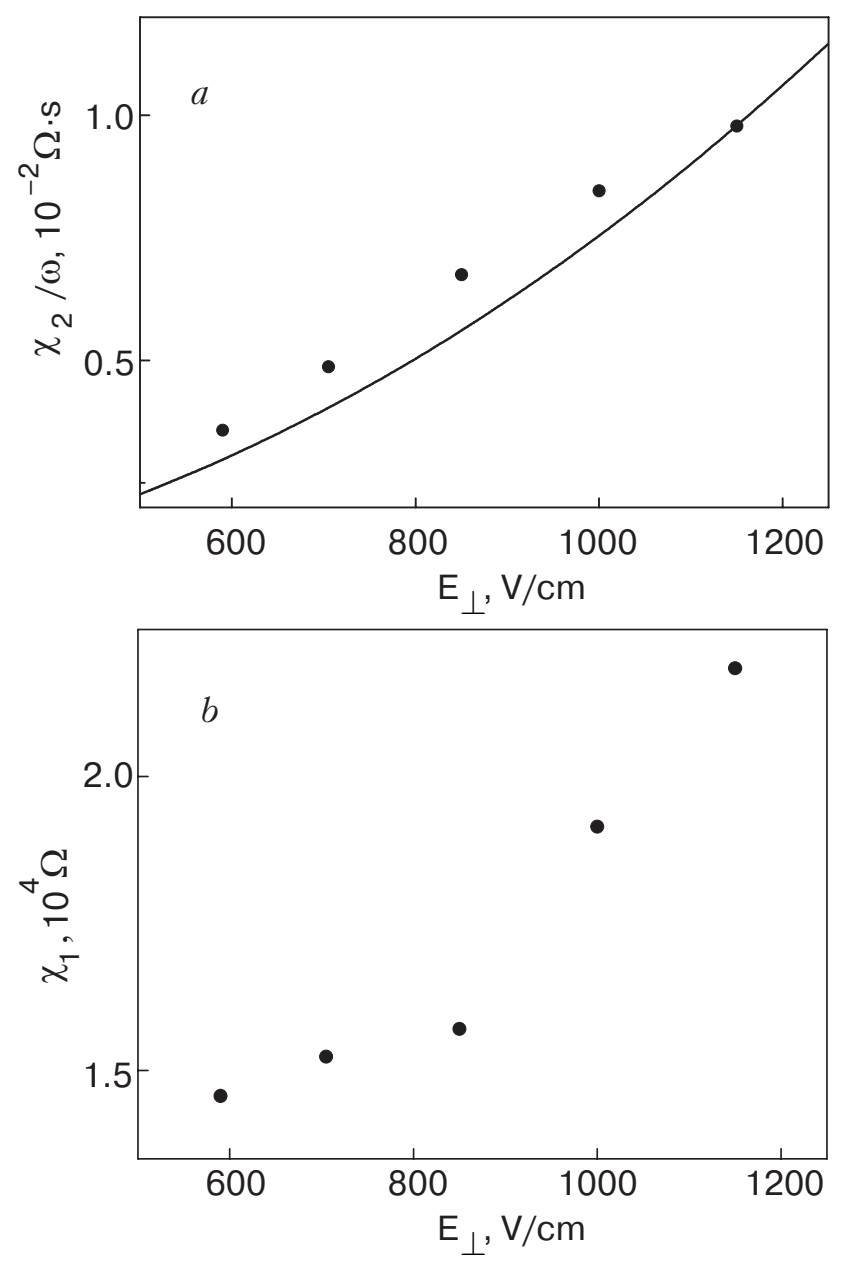

Fig. 2. Holding-field dependences of the inverse conductivity of the electron crystal measured at the resonance frequency: imaginary part $(a)$ and real part $(b)$. The solid line in Fig. 2, $a$ corresponds to the theory (see text).

In present work for the calculation of $\chi_{1}$ and $\chi_{2}$ we used the amplitude and phase shift of the induced current at a fixed frequency. The most convenient for the calculations are the measurements at the resonance frequency because of the highest signal-to-noise ratio and suppressed influence of errors, especially systematic errors of measurement. However, the probability of nonlinear processes in the EC is present at the resonance because the leading electric field can reach high values. The problem of the influence of the leading fields needs a special analysis and is not discussed in the present report.

One can express the relation between $\chi_{1}$ and $\chi_{2}$ and the components of $Z_{1}$ calculated in Ref. 6:

$$
\chi_{1}=\frac{m \omega}{n e^{2}} \operatorname{Im} Z_{1}, \chi_{2}=-\frac{m \omega}{n e^{2}} \operatorname{Re} Z_{1} .
$$

Although experimental conditions and the theoretical model are not the same, the theory is rather general and is supposed to reflect adequately the main fea- tures of our experiment. Without rewriting the complete expression [6], let us note that at low enough frequencies $\left(\omega<\omega_{1}\right.$, where $\omega_{1}$ is the lowest capillary wave mode) $\operatorname{Re} Z_{1} \sim C_{1}$ and $\operatorname{Im} Z_{1} \sim C_{1} \gamma / \omega$, where $C_{1}$ is the electron-ripplon interaction coefficient, which depends on the electron density, temperature, holding electric field etc., and $\gamma$ is the capillary wave attenuation coefficient. It is clear that $\chi_{2} / \omega \sim \operatorname{Re} Z_{1}$ and $\chi_{1} \sim \operatorname{Im} Z_{1}$. In Figure 2 the holding field dependences of $\chi_{2} / \omega$ and $\chi_{1}$ are shown. The solid line in Fig. 2, $a$ is the theoretical prediction for the dependence. The theoretical calculations do not contain any fitting or scale coefficient and lead to a good consistency with the experiment.

The real part of the inverse conductivity $\chi_{1}$ increases with increasing $E_{\perp}$ approximately as $E_{\perp}^{2}$ (Fig. 2,b). That is in accordance with the theory [6] because of $C_{1} \sim E_{\perp}^{2}$, but the value of $\chi_{1}$ is much higher that the theoretical estimations considered capillary wave attenuation as the main mechanism for dissipation. A reason for the high observed dissipation remains unclear.

The change of the resonance spectrum with increasing $E_{\perp}$ probably (Fig. 1,a) reflects the appearance of nonlinearity in the crystal response to the leading field, and it is quite possible that the interaction of ripplons with structural defects in the EC can play an essential role in energy losses in the crystal. In this can onnection additional studies of the influence of the leading field on the processes in ECs over liquid helium are highly desirable.

We would like to thank V. Dotsenko for help in the experiments.

1. C.C. Grimes and G. Adams, Phys. Rev. Lett. 42, 795 (1979).

2. D.S. Fisher, B.I. Galperin, and P.M. Platzman, Phys. Rev. Lett. 42, 798 (1979).

3. Yu.P. Monarkha and V.B.Shikin, Fiz. Nizk. Temp. 9, 913 (1983) [Sov. J. Low Temp. Phys. 9, 471 (1983)].

4. V.E. Sivokon, V.V. Dotsenko, Yu.Z. Kovdrya, and V.N. Grigor'ev, Fiz. Nizk. Temp. 22, 1107 (1996) [Low Temp. Phys. 22, 845 (1996)].

5. G. Deville, Phys. Rev. Lett. 72, 135 (1988).

6. Yu.P. Monarkha, Fiz. Nizk. Temp. 6, 685 (1980) [Low Temp. Phys. 6, 331 (1980)].

7. Yu.P. Monarkha, Fiz. Nizk. Temp. 7, 692 (1981) [Sov. J. Low Temp. Phys. 7, 338 (1981)].

8. V.V. Dotsenko, V.E. Sivokon, Yu.Z. Kovdrya, and V.N. Grigor'ev, Fiz. Nizk. Temp. 23, 1028 (1997) [Sov. J. Low Temp. Phys. B22, 772 (1997)].

9. V.E. Sivokon, V.V. Dotsenko, S.S. Sokolov, Yu.Z. Kovdrya, and V.N. Grigor'ev, Fiz. Nizk. Temp. 22, 715 (1996) [Low Temp. Phys. 22, 549 (1996)].. 